\title{
Caracterização espectral de sinais caóticos
}

\author{
Daniela Mitie Kato e Marcio Eisencraft
}

\begin{abstract}
Resumo-Este artigo investiga numericamente as características da Densidade Espectral de Potência (DEP) de sinais caóticos gerados por mapas da família tenda inclinada. $A$ influência do expoente de Lyapunov na sequiência de autocorrelação e na DEP é avaliada por meio de simulações computacionais. Conclui-se que a banda essencial está diretamente relacionada a esse expoente e que estes sinais podem ser passa-altas ou passabaixas, dependendo do parâmetro da família. Estes resultados são relevantes para aplicações de sinais caóticos em sistemas de modulação digital.
\end{abstract}

Palavras-Chave-Comunicações usando caos, geradores caóticos, analise espectral.

Abstract-This paper numerically investigates characteristics of the Power Spectral Density (PSD) of chaotic signals generated by skew tent maps. The influence of the Lyapunov exponent on the autocorrelation sequence and on the PSD is evaluated via computational simulations. We conclude that the essential bandwidth is strongly related to this exponent and these signals can be low-pass or high-pass depending on the family's parameter. These results are relevant when it comes to applications of chaotic signals in digital modulation systems.

Keywords - Chaotic communication, chaos generators, spectral analysis.

\section{INTRODUÇÃO}

Sistemas dinâmicos discretos unidimensionais são modelos matemáticos em que o estado atual é dependente do estado anterior. A função que relaciona esses estados é chamada de mapa [1]. Neste artigo, estudam-se numericamente características espectrais de sinais caóticos gerados por mapas unidimensionais.

Um sinal caótico é aperiódico, determinístico e apresenta Dependência Sensível às Condições Iniciais (DSCI). A DSCI significa que, para duas condições iniciais muito próximas, a cada iteração do mapa, os sinais resultantes vão se distanciando, tornando-se totalmente distintos [1]. Apesar de sua complexidade, estes sinais podem ser facilmente obtidos a partir de sistemas dinâmicos discretos unidimensionais.

Devido às propriedades que os definem, costuma-se afirmar que os sinais caóticos, caracteristicamente, ocupam uma larga faixa de freqüências, possuem seqüência de autocorrelação impulsiva e que as seqüências de correlação cruzada entre sinais com diferentes condições iniciais apresentam valores baixos [2].

Atualmente, existe um grande número de áreas desenvolvendo pesquisas com a aplicação de sinais caóticos [3], [4]. Na Engenharia de Telecomunicações não é diferente. A partir do trabalho [5], vêm surgindo numerosas possibilidades de aplicação desses sinais em modulação analógica e digital,

Daniela Mitie Kato e Marcio Eisencraft, Escola de Engenharia, Universidade Presbiteriana Mackenzie, São Paulo, Brasil, e-mails: danikato@yahoo.com, marcioft@mackenzie.br. codificação e criptografia, entre outras áreas. Veja, por exemplo, [2], [6] e suas referências.

Um exemplo interessante de aplicação dos sinais caóticos é no Espalhamento Espectral por Seqüência Direta ( $D S-S S$ - Direct Sequence - Spread Spectrum), em que podem ser aplicados como seqüências espalhadoras. Sendo sinais banda larga e com DSCI, é possível gerar infinitas sequiências com baixo custo [2].

Apesar da grande quantidade de trabalhos publicados, poucos abordam as características espectrais dos sinais caóticos. Alguns trabalhos, como [7]-[11], mostram gráficos da Densidade Espectral de Potência (DEP) de sinais caóticos de tempo contínuo gerados por sistemas particulares. $\mathrm{O}$ artigo [12] utiliza análise da DEP de sinais caóticos de tempo discreto para estudar intermitências. Porém, um estudo mais aprofundado das características da DEP de sinais caóticos, fator fundamental para a utilização prática desses sinais em Telecomunicações, ainda é necessário.

O objetivo deste trabalho é apresentar alguns resultados preliminares sobre as características espectrais de sinais caóticos gerados pela família de mapas tenda inclinada. Além disso, busca-se relacionar essas características com uma importante medida da "caoticidade": o expoente de Lyapunov. Vale ressaltar que estas avaliações foram realizadas de forma empírica e as conclusões são baseadas em simulações computacionais. Esta abordagem, comum no estudo de aplicações de sinais caóticos, é justificada pela dificuldade em se obter resultados analíticos quando se estuda sistemas intrinsecamente não-lineares, como os em questão.

Um mapa tenda inclinada [13] é composto por dois trechos lineares, com inclinações de sinais diferentes. Eles se encontram em um ponto que determina o pico da tenda. A abscissa desse ponto é o parâmetro que define um mapa na família. Os sinais gerados por estes mapas apresentam comportamento rico e variado, mas ainda assim de fácil esquematização. Daí a escolha desta família nestes estudos iniciais.

Este trabalho está organizado da seguinte forma: na Seção II é introduzida a família de mapas tenda inclinada e suas principais características. Na Seção III são discutidas as técnicas de obtenção da DEP de sinais caóticos. Na Seção IV é analisada a relação entre banda essencial e expoente de Lyapunov. Por fim, na Seção V, são expostas as conclusões do trabalho.

\section{A FAmília de Mapas TEnda InClinadA}

Um sistema dinâmico unidimensional em tempo discreto é definido pela equação de diferenças

$$
s(n+1)=f(s(n)),
$$

sendo $U \subset \mathbb{R}$ o domínio de $f(s), n \in \mathbb{N}$ e $s(0) \in U$. Para cada condição inicial $s(0)=s_{0}$, uma órbita ou sinal $s\left(n, s_{0}\right)$ 

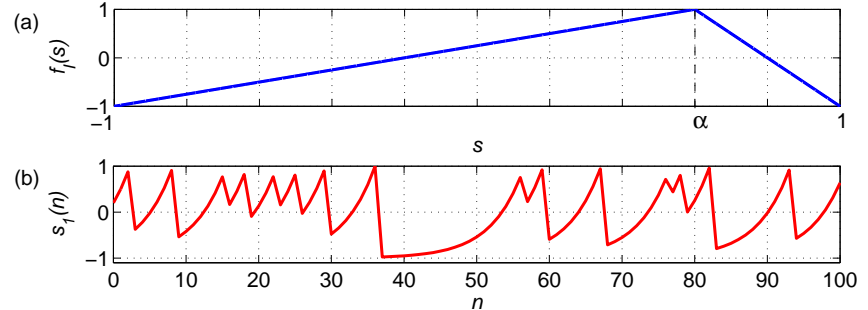

(c)

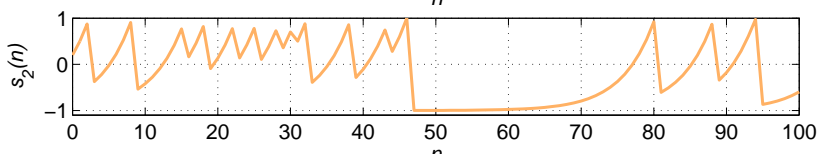

(d)

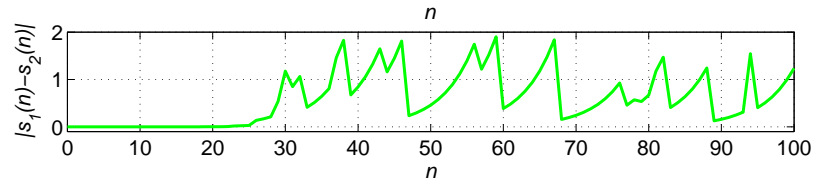

Fig. 1. (a) Mapa tenda inclinada, (b) órbita do mapa tenda inclinada $s_{1}(n)$ com $s_{0}=0.2$, (c) órbita do mapa tenda inclinada $s_{2}(n)$ com $s_{0}=0.2000001$ e (d) modulo da diferença entre as órbitas $s_{1}(n)$ e $s_{2}(n)$.

é definido. Quando a condição inicial $s_{0}$ estiver subentendida ou não for relevante, $s\left(n, s_{0}\right)$ é representado simplesmente por $s(n)$.

Neste trabalho, foca-se na família de mapas tenda inclinada, uma modificação do mapa proposto em [13]. Um mapa desta família é definido por

$$
s(n+1)=f_{I}(s(n))
$$

em que

$$
f_{I}(s)= \begin{cases}\frac{2}{\alpha+1} s+\frac{1-\alpha}{\alpha+1}, & -1<s<\alpha \\ \frac{2}{\alpha-1} s-\frac{\alpha+1}{\alpha-1}, & \alpha \leq s<1\end{cases}
$$

e $\{\alpha, s(0)\} \subset U=(-1,1)$. O parâmetro $\alpha$ é a abscissa em que se localiza o ápice da tenda.

Na Figura 1(a) é ilustrado um exemplo de mapa tenda inclinada para $\alpha=0.6$. Na Figura 1(b) um sinal $s_{1}(n)$ deste mapa com condição inicial $s_{0}=0.2$ é mostrado. $\mathrm{Na}$ Figura 1(c) é apresentado outro sinal gerado por este mapa, $s_{2}(n)$, com $s_{0}=0.2000001$, ilustrando o conceito de DCSI característico de sinais caóticos. Após cerca de 30 iterações, a diferença entre os sinais fica da ordem de grandeza dos próprios sinais, como mostrado na Figura 1(d).

A DSCI é geralmente verificada por meio do expoente de Lyapunov. Este expoente mede a taxa de divergência exponencial média entre duas órbitas muito próximas. Se o valor do expoente é maior que zero, então um sinal aperiódico é caótico [1].

O expoente de Lyapunov $h$ de uma órbita $s\left(n, s_{0}\right)$ é calculado por meio de [1]

$$
h=\lim _{N \rightarrow \infty} \frac{1}{N}\left(\sum_{n=0}^{N-1} \ln \left|f^{\prime}\left(s\left(n, s_{0}\right)\right)\right|\right),
$$

em que $f^{\prime}(s)$ é a derivada de $f(s)$.

Pode-se mostrar que, para as órbitas da família de mapas tenda inclinada, o expoente de Lyapunov depende apenas do

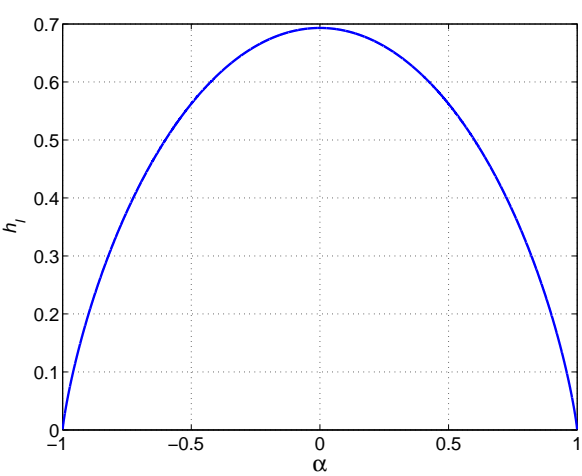

Fig. 2. Expoente de Lyapunov para $\alpha$ no intervalo $(-1,1)$.

parâmetro $\alpha$, sendo dado por [13]

$$
h_{I}=\frac{\alpha+1}{2} \ln \left(\frac{2}{\alpha+1}\right)+\frac{1-\alpha}{2} \ln \left(\frac{2}{1-\alpha}\right) .
$$

Na Figura 2 é mostrado um gráfico de $h_{I}$ em função de $\alpha$. Nota-se que, para todos os valores de $\alpha$ contidos no intervalo $(-1,1)$, o expoente de Lyapunov é positivo. Isto implica que o mapa gera sinais caóticos para todos os valores admissíveis de $\alpha$. O máximo valor de $h_{I}$ é ln 2 para $\alpha=0$.

Os mapas $f_{I}(s)$ possuem densidade invariante uniforme independentemente do parâmetro $\alpha$ [14]. Isto significa que os pontos dos sinais gerados por estes mapas distribuem-se uniformemente no intervalo $U=(-1,1)$. Conseqüentemente, esses sinais possuem média nula e potência média de $1 / 3$ para qualquer valor de $\alpha$ [14].

\section{Densidade Espectral de PotênCIA}

Os sinais caóticos gerados por um mapa podem ser analisados de duas maneiras: (i) como sinais individuais determinísticos ou (ii) como funções amostra de um processo estocástico. Essas duas maneiras levam a diferentes formas do cálculo da DEP, que são analisadas nesta seção.

\section{A. Sinais Individuais Determinísticos}

Dada a Eq. (2) e a condição inicial $s(0)=s_{0}$, o sinal $s\left(n, s_{0}\right)$ fica bem definido. Desta forma, pode-se calcular sua seqüência de autocorrelação $R\left(l, s_{0}\right)$ como

$$
R\left(l, s_{0}\right)=\lim _{N \rightarrow \infty} \frac{1}{N} \sum_{n=0}^{N-1} s\left(n, s_{0}\right) s\left(n+l, s_{0}\right),
$$

em que $l$ é um número inteiro [15]. Caso $n+l<0$, assume-se que $s\left(n+l, s_{0}\right)=0$. Explicita-se na notação a dependência da seqüência de autocorrelação com a particular condição inicial $s_{0}$.

A DEP $S\left(f, s_{0}\right)$ é obtida calculando-se a Transformada de Fourier de Tempo Discreto (TFTD) de $R\left(l, s_{0}\right)$, considerandose $l$ a variável temporal [15].

Na Figura 3 são ilustrados seis trechos de diferentes órbitas geradas usando as Eqs. (2) e (3) e suas respectivas DEPs. Para a estimação da DEP foram utilizadas 10000 iterações, sendo descartadas as 200 primeiras, resultando em $N=9800$ amostras. Foram usados $\alpha=-0.9, \alpha=0$ e $\alpha=0.9$ e duas 
(a)

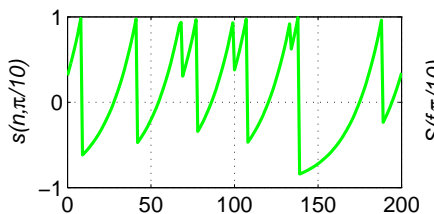

(b)

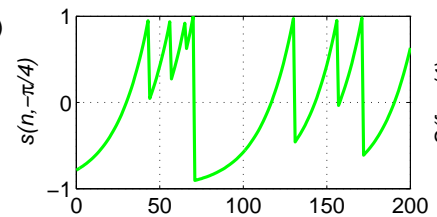

(c)

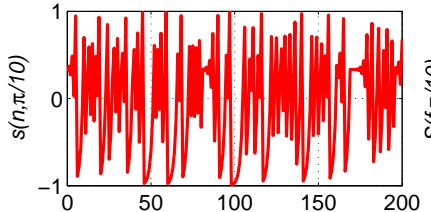

(d)
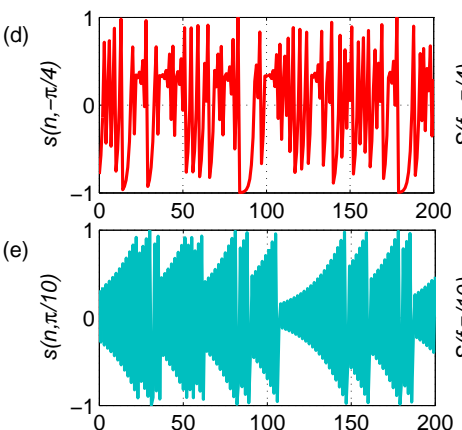

(f)

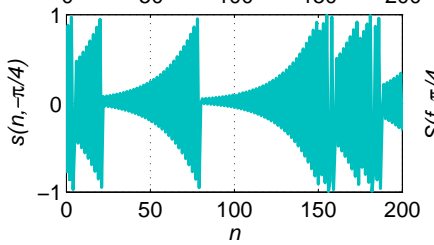

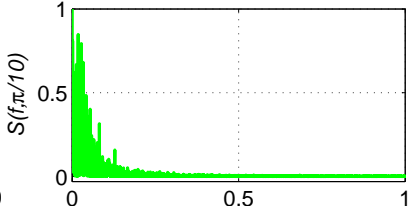
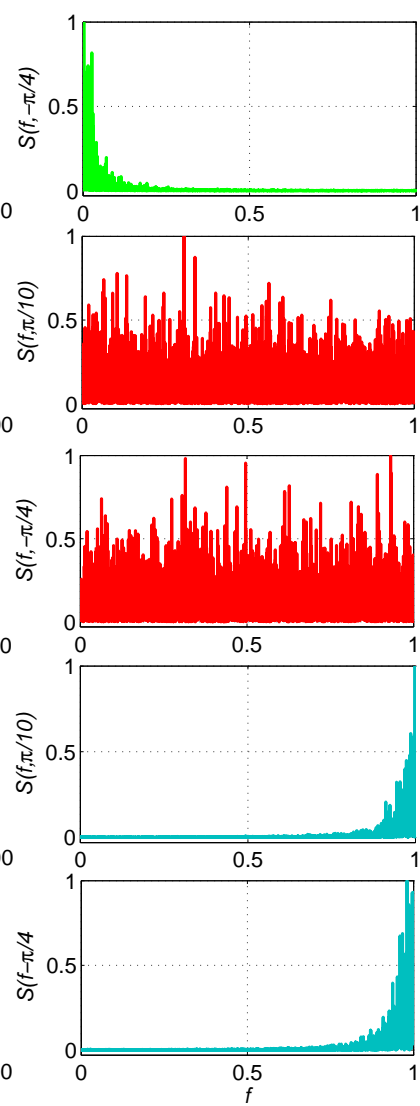

Fig. 3. Sinais gerados pelo mapa tenda inclinada e suas respectivas DEPs para (a) $s_{0}=\pi / 10, \alpha=0.9$; (b) $s_{0}=-\pi / 4, \alpha=0.9$; (c) $s_{0}=\pi / 10, \alpha=0$; (d) $s_{0}=-\pi / 4, \alpha=0$; (e) $s_{0}=\pi / 10, \alpha=-0.9$ e; (f) $s_{0}=-\pi / 4$, $\alpha=-0.9$.

condições iniciais para cada um desses valores. É importante citar que o mapa com $\alpha=0$ gera órbitas caóticas apenas para condições iniciais irracionais [1]. Sendo assim, apenas neste caso, utilizou-se a propriedade da conjugação [1] deste mapa com o mapa quadrático

$$
f_{Q}(s(n))=-2 s^{2}(n)+1 .
$$

Nos gráficos das DEPs, tanto a abscissa quanto a ordenada estão normalizadas. Desta maneira, $f=1$ equivale a $\omega=\pi$ $\mathrm{rad} / \mathrm{amostra}$ no tempo discreto e a $f_{c}=f_{a} / 2$, em que $f_{a}$ é a freqüência de amostragem, no tempo continuo.

Por meio das simulações computacionais realizadas, podese inferir que:

i) para $\alpha$ positivo, os sinais se comportam como passabaixas e a oscilação no tempo é lenta, como se observa, por exemplo, nas Figuras 3(a) e 3(b);

ii) para $\alpha$ próximo de zero, os sinais são banda larga, como pode ser visto nas Figuras 3(c) e 3(d), e

iii) para $\alpha$ negativo, os sinais se comportam como passaaltas e a oscilação no tempo é rápida, como é ilustrado
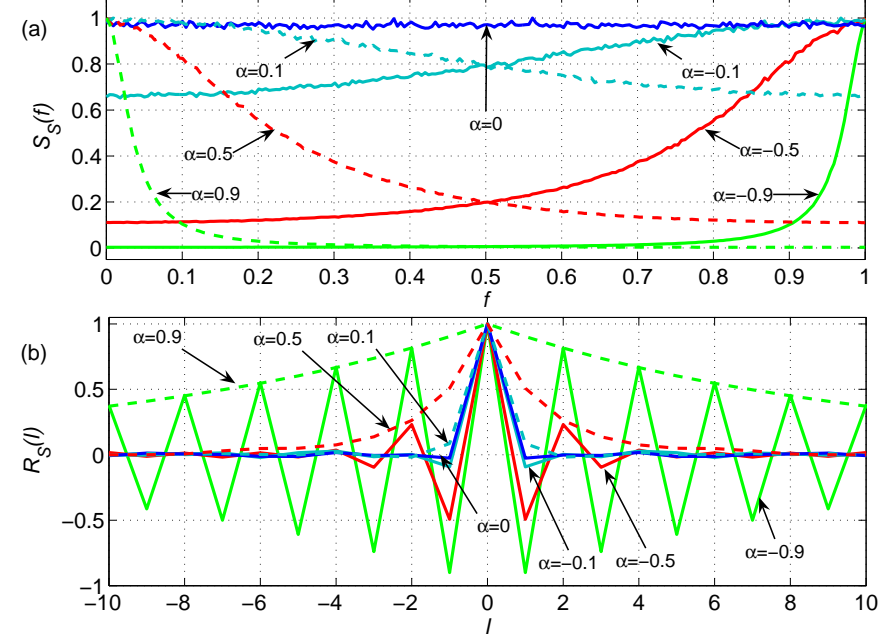

Fig. 4. (a) DEP e (b) seqüência de autocorrelação para sinais do mapa tenda inclinada para alguns valores de $\alpha$.

nas Figuras 3(e) e 3(f).

Além disso, condições iniciais diferentes para um mesmo valor de $\alpha$ não alteram significativamente as características no domínio da freqüência. $\mathrm{O}$ fato das características espectrais não dependerem das condições iniciais, leva à idéia de se modelar um sinal caótico como uma função amostra de um processo aleatório ergódico, definido pelo seu mapa gerador. Essa abordagem é discutida a seguir.

\section{B. Funções Amostra de Um Processo Estocástico}

Os sinais caóticos podem ser compreendidos como um processo estocástico, em que cada sinal gerado por uma condição inicial diferente representa uma função amostra. Neste caso, o mapa define um processo ergódico [16] e sua seqüência de autocorrelação é definida por

$$
R_{S}(l)=E\left[R\left(l, s_{0}\right)\right],
$$

em que $E[\cdot]$ é o operador esperança matemática. Essa esperança é tomada sobre todas as condições iniciais que geram órbitas caóticas. A DEP $S_{S}(f)$ é a TFTD de $R_{S}(l)$ [17], como é feito usualmente em processos estocásticos convencionais.

Na Figura 4 são ilustradas estimativas da DEP e da sequiência de autocorrelação normalizadas para diferentes valores de $\alpha$, utilizando 20000 órbitas com condições iniciais $s_{0}$ distribuídas uniformemente no domínio $U$, cada uma com $N=440$ amostras. Utilizar um valor maior de $N$ não altera numericamente os resultados obtidos. Na Figura 5 é ilustrado o comportamento geral dessas funções para valores de $\alpha$ no intervalo $(-1,1)$.

Analisando essas figuras, observa-se que:

i) quanto maior o módulo de $\alpha$, mais estreita é a banda dos sinais resultantes;

ii) o sinal de $\alpha$ define se as órbitas geradas têm comportamento passa-baixas ou passa-altas;

iii) as DEPs de sinais gerados por mapas com valores de $\alpha$ opostos apresentam simetria em relação a $f=0.5$, como pode ser visto na Figura 4(a); 

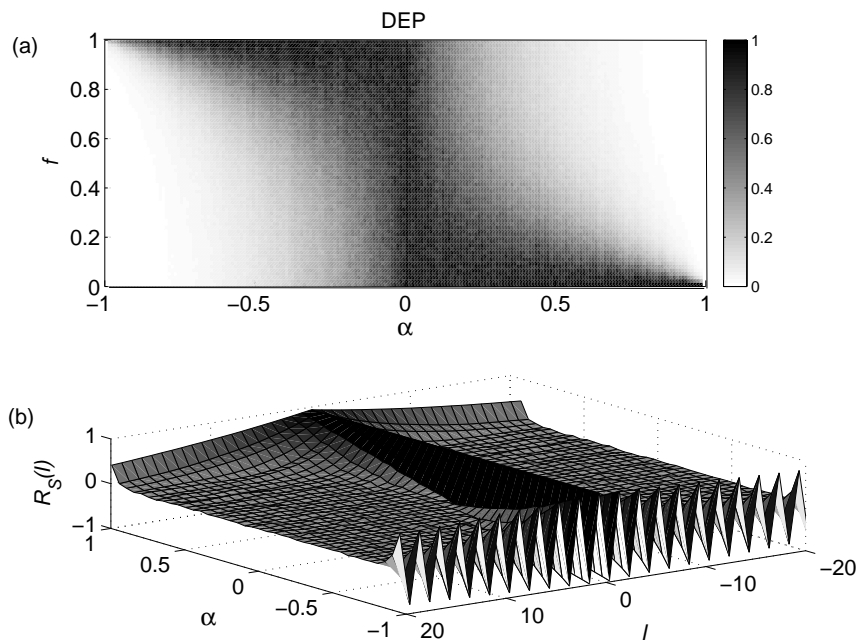

Fig. 5. (a) DEP e (b) seqüência de autocorrelação para sinais do mapa tenda inclinada para valores de $\alpha$ no intervalo $(-1,1)$.

iv) para $\alpha>0, R_{S}(l)$ decai monotonicamente com $|l|$, e para $\alpha<0, R_{S}(l)$ oscila, indicando que, neste caso, para quase quaisquer $n$ e $s_{0}$, as amostras $s\left(n, s_{0}\right)$ e $s(n+$ $\left.1, s_{0}\right)$ têm sinais diferentes.

Estes resultados podem ser melhor quantificados utilizandose o conceito de banda essencial.

\section{BANDA ESSENCIAL}

A banda essencial $B$ é definida como a largura de banda em que $95 \%$ da potência do sinal está concentrada [18]. Neste trabalho, a banda essencial foi dividida por 0.95 , sendo apresentada de forma normalizada. Assim, um ruído branco, cuja potência está distribuída uniformemente por todo o espectro, possui $B=1$. Para qualquer outro processo, $0<B<1$.

Pela Eq. (5), o valor de $|\alpha|$ define o expoente de Lyapunov. Na Figura 4(a), vê-se que este parâmetro está diretamente relacionado à largura de banda do sinal. Conseqüentemente, é possível relacionar a banda essencial com o parâmetro $\alpha$ e com o expoente de Lyapunov, como ilustrado na Figura 6.

No gráfico 6(a), constata-se que se obtém um processo ruído branco para $\alpha=0$ e um processo de banda extremamente estreita para $\alpha \approx 1$. É importante ressaltar que, em todos os casos, o expoente de Lyapunov é positivo e assim, as funçõesamostra aperiódicas destes processos são sinais caóticos. Esta relação entre $B$ e $|\alpha|$ é justificada pelo fato de que, observando a Figura 2, quanto menor $|\alpha|$, maior o valor do expoente de Lyapunov, o que significa que os sinais com condições iniciais próximas divergem mais rapidamente. Desta maneira, os sinais se tornam totalmente distintos após poucas iterações, levando a uma seqüência de autocorrelação impulsiva. Sendo a DEP calculada pela TFTD da sequiência de autocorrelação, tem-se um sinal banda larga, ou seja, com $B$ elevado.

Assim, escolhendo-se um valor de $\alpha$ adequado, pode-se obter um sinal caótico banda larga ou estreita, passa-altas ou passa-baixas e com a banda essencial desejada.

Estes resultados contrariam o que se costuma afirmar sobre os sinais caóticos — que ocupam largas faixas de freqüência

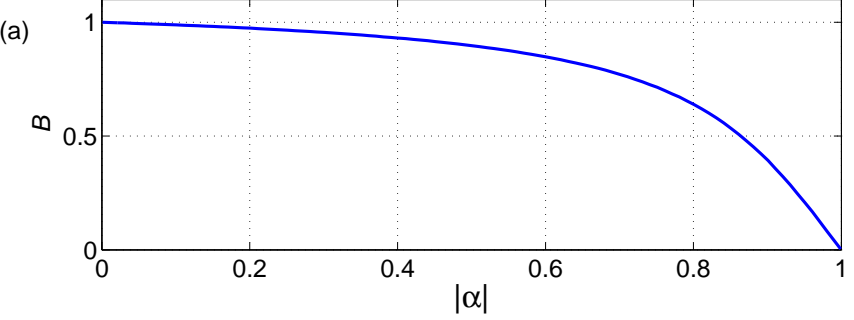

(b)

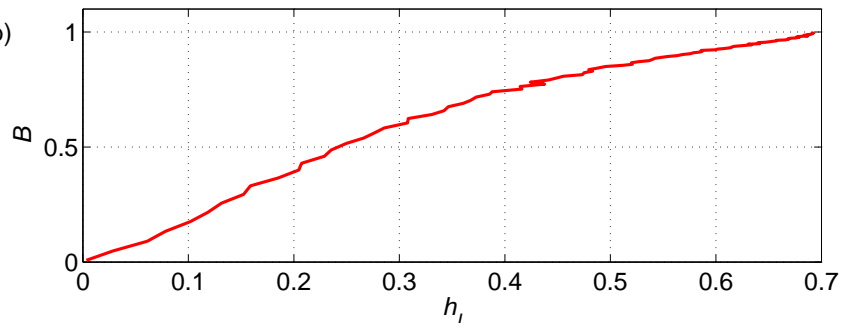

Fig. 6. Banda essencial em função (a) de $|\alpha|$ e (b) do expoente de Lyapunov.

e que sua função de autocorrelação é representada na forma de um impulso.

Por isso é importante estudar mais profundamente o espectro desses sinais pensando em aplicá-los em Telecomunicações.

\section{CONCLUSÕES}

Com as características usualmente conhecidas dos sinais caóticos, é possível aplicá-los em diversas áreas da Engenharia de Telecomunicações, como discutido na Seção I.

Com a realização deste trabalho, mostrou-se, por meio de simulações computacionais, a possibilidade de se gerar sinais caóticos banda estreita, com banda essencial bem definida, sem prejuízo na "caoticidade". Além disso, eles podem apresentar seqüência de autocorrelação não impulsiva.

Notou-se também que o parâmetro $\alpha$ e o expoente de Lyapunov estão fortemente relacionados com a banda essencial $B$. Desta maneira, tendo uma banda essencial desejada, facilmente pode-se obter $\alpha$ e um mapa linear por partes associado que gera esta órbita.

Estuda-se atualmente a possibilidade de que as características espectrais de sinais caóticos gerados por um determinado mapa, sejam expandidas para sinais gerados por um mapa conjugado a este. A conjugação relaciona um sinal de um determinado mapa ao sinal de um outro mapa por meio de uma função bijetora [1]. Assim, os resultados apresentados aqui poderão ser generalizados para outros mapas unidimensionais.

O ponto mais relevante observado neste trabalho, é que caos não é sinônimo de banda larga e seu espectro necessita de um estudo mais aprofundado, quando se pensa em aplicações práticas dos mesmos na área de Telecomunicações.

\section{Agradecimentos}

Os autores gostariam de agradecer ao apoio financeiro recebido do MackPesquisa (fundo de incentivo à pesquisa do Instituto Presbiteriano Mackenzie). Agradecem também à Profa. Maria D. Miranda e ao Prof. José R. C. Piqueira pelas motivantes discussões sobre os resultados obtidos neste trabalho. 


\section{REFERÊNCIAS}

[1] K. T. Alligood, T. D. Sauer and J. A. Yorke, Chaos - an introduction to dynamical systems. Springer, 1996.

[2] F. C. M. Lau and C. K. Tse, Chaos-based digital communication systems. Springer, 2003.

[3] S. H. Strogatz, Nonlinear dynamics and chaos with applications to physics, biology, chemistry and engineering. Addinson-Wesley, 1998.

[4] L. H. A. Monteiro, Sistemas dinâmicos. Livraria da Física, 2006.

[5] L. M. Pecora and T. L. Carroll, "Synchronization in chaotic systems," Physical Review Letters, v. 64, n. 8, p. 821-824, Fevereiro 1990.

[6] M. P. Kennedy, R. Rovatti and G. Setti, Chaotic electronics in telecommunications. CRC Press, 2000.

[7] T. Matsumoto and L. O. Chua, "Hyper chaos: laboratory experiment and numerical confirmation," IEEE Transactions on Circuits and Systems I, v. 33, n. 11, p. 1143-1147, Novembro 1986.

[8] T. Matsumoto, L. O. Chua and M. Komuro, "The double scroll," IEEE Transactions on Circuits and Systems - I, v. 32, n. 8, p. 798-817, Agosto 1985.

[9] K. M. Short, "Steps toward unmasking secure communications," International Journal of Bifurcation and Chaos, v. 4, n. 4, p. 959-977, Fevereiro 1994.

[10] T. El Assad and C. Tarhini, "Design and implementation of a chaotic codec," Proc. European Conference on Wireless Technology, p. 351-354, Outubro 2005.

[11] R. R. C. Reddy, T. Srinivas and A. Nadarajan, "Comparative study between multi level modulations in chaotic optical communications," Proc. IFIP International Wireless and Optical Communication Networks, Abril 2006.

[12] K. Ullmann and I. L. Caldas, "Transitions in the parameter space of a periodically forced dissipative system," Chaos, Solitons \& Fractals, v. 7, n. 11, p. 1913-1921, Novembro 1996.

[13] A. Kisel, H. Dedieu and T. Schimming, "Maximum likelihood approaches for noncoherent communications with chaotic carriers," IEEE Transactions Circuits and Systems - I, v. 48, n. 5, p. 533-542, Maio 2001.

[14] M. Eisencraft, Contribuições da teoria da estimação para modulações digitais que utilizam sinais caóticos. Tese - (Doutorado em Engenharia Elétrica) - Escola Politécnica da Universidade de São Paulo, 2006.

[15] S. K. Mitra, Digital signal processing: a computer-based approach. McGraw-Hill, 2006.

[16] A. Lasota and M. Mackey, Probabilistic properties of deterministic systems. Cambridge University, 1985.

[17] S. S. Haykin, Communication Systems. Wiley, 2000.

[18] B. P. Lathi, Modern digital and analog communication systems. Oxford University Press, 1998. 